\title{
ISIEA: An image database of social inclusion and exclusion in young Asian adults
}

\author{
Zixin Zheng ${ }^{1,2,3} \cdot$ Sijin $\mathrm{Li}^{1,2,3} \cdot$ Licheng Mo ${ }^{1,2,3} \cdot$ Weimao Chen ${ }^{1,2,3} \cdot$ Dandan Zhang ${ }^{1,2,3}$ (1)
}

Accepted: 26 October 2021 / Published online: 16 December 2021

(c) The Author(s) 2021

\begin{abstract}
Human beings have a fundamental need to belong. Evaluating and dealing with social exclusion and social inclusion events, which represent negative and positive social interactions, respectively, are closely linked to our physical and mental health. In addition to traditional paradigms that simulate scenarios of social interaction, images are utilized as effective visual stimuli for research on socio-emotional processing and regulation. Since the current mainstream emotional image database lacks social stimuli based on a specific social context, we introduced an open-access image database of social inclusion/ exclusion in young Asian adults (ISIEA). This database contains a set of 164 images depicting social interaction scenarios under three categories of social contexts (social exclusion, social neutral, and social inclusion). All images were normatively rated on valence, arousal, inclusion score, and vicarious feeling by 150 participants in Study 1. We additionally examined the relationships between image ratings and the potential factors influencing ratings. The importance of facial expression and social context in the image rating of ISIEA was examined in Study 2. We believe that this database allows researchers to select appropriate materials for socially related studies and to flexibly conduct experimental control.
\end{abstract}

Keywords Social exclusion $\cdot$ Social inclusion $\cdot$ Ostracism $\cdot$ Image database $\cdot$ Social cognition $\cdot$ Social reward $\cdot$ Social punishment

\section{Introduction}

Complex social interactions pervade our daily lives because of human beings' social nature. As a fundamental human need, the need to belong strongly drives people's desire to establish and maintain interpersonal relationships with others (Baumeister \& Leary, 1995). Whether belonging needs are satisfied has a significant impact on our physical and mental health (Baumeister \& Leary, 1995). Positive social ties, such as being socially included, accepted, or supported, have been widely observed to be associated with pleasant feelings, healthy physiological functions, and good physical health (Kiecolt-Glaser et al., 2010; Eisenberger, 2003, 2013;

Dandan Zhang

zhangdd05@gmail.com

1 School of Psychology, Shenzhen University, Shenzhen 518060, China

2 Magnetic Resonance Imaging (MRI) Center, Shenzhen University, Shenzhen 518060, China

3 Shenzhen-Hong Kong Institute of Brain Science, Shenzhen 518055, China
Pressman, 2019). Some researchers have suggested that positive emotions, positive social connections, and physical health influence each other in a self-sustaining upward-spiral dynamic (Kok et al., 2013). Conversely, social pain refers to a painful experience associated with actual or potential damage to desired social connections (Eisenberger, 2012, 2015), usually caused by social exclusion or rejection. Previous studies have found that social exclusion induces negative or painful emotions and threatens a human's fundamental needs (Williams, 2007).

Traditional studies on social exclusion/inclusion generally use paradigms, such as cyberball (Williams et al., $2000 ; 2003)$, to simulate a social interaction scenario. The image-based imagination paradigm also serves as an effective way to examine the social interaction context (Elliott et al., 2012; Zhao et al., 2021). Humans can experience the empathic feeling of others' emotions by simulating what is happening in the minds of who they are observing (Singer \& Lamm, 2009; Keysers \& Gazzola, 2007). Studies have demonstrated that participants have similar behavioral and neural responses observed in the cyberball game when they view images of social exclusion situations (e.g., the activation of 
dorsal anterior cingulate cortex; Kross et al., 2007; Premkumar et al., 2012; Eisenberger, 2015). Meanwhile, other studies have found that simply viewing images of social support figures (e.g., romantic partners) can reduce painful experiences, supported by both self-reported and neural activity data (Eisenberger et al., 2011; Younger et al., 2010). Importantly, in a study investigating the neuronal deficits of social stimuli processing in individuals with major depression, Elliott et al. (2012) collected a set of images of social exclusion and inclusion scenarios and asked participants to imagine themselves as the highlighted person in the picture and to experience their affective feelings. Inspired by this study, our lab has performed a series of studies, using a set of exclusion images and asked participants to imagine feeling the emotions of the highlighted person in the images (He et al., 2018; 2020a, b; Zhao et al., 2021). These images were verified to be effective in inducing social pain, which helped us study the emotional regulation effect in response to social pain.

Although the image-based imagination paradigm may not elicit emotions as real as "first-hand" pain-inducing paradigms, such as the cyberball game, it contributes to social cognition research with its own advantages. Using images as stimuli is especially suitable for event-related designed neuroscience experiments that require precise time accuracy and an adequate number of trials for reliable signals (e.g., fMRI signals and event-related potentials). Moreover, image viewing is a simple and understandable task for specific participants, such as children and psychiatric patients, and this paradigm does not elicit excessively negative emotions that might hurt these participants (Singer \& Lamm, 2009). Given these features and empirical evidence supporting the validity of the imagination paradigm and social image stimuli, a set of standardized images is urgently needed for research and clinical practice.

Images are widely used as effective visual stimuli in affective research. To date, there have been many emotionalimage databases with ratings of emotional characteristics (Barrett, 2006; Dalgleish, 2004; Ekman, 1992). The most popular databases include the International Affective Image System (IAPS; Lang et al., 1999), the Geneva Affective Image Database (GAPED; Dan-Glauser \& Scherer, 2011), and the Nencki Affective Image System (NAPS; Marchewka et al., 2014). These databases covered various themes of images, including people, animals, objects, and natural scenes. However, limited image databases have been identified in the field of social interactions. Some image databases provide social stimuli using isolated faces without social contextual information (e.g., the Chicago Face Database; Ma et al., 2015). The Pictures with Social Context and Emotional Scenes (PiSCES; Teh et al., 2018) is a valuable database that provides black-and-white line drawings depicting various social situations. However, it lacks a detailed classification of complex social situations, so it could not meet the need to examine specific social conditions, such as social inclusion and exclusion. Additionally, we think that line drawings decrease the realness and ecological validity of social interaction scenarios. Besides these databases, some studies used social inclusion/exclusion images collected from the internet (e.g., Elliott et al. (2012) used eight negative and eight positive social interaction images), but these materials are usually not open-access due to issues such as copyrights and the ratings of the emotional characteristics of each image were not provided. Due to limited image databases in the field of social interaction, especially social inclusion/exclusion, researchers must perform timeconsuming additional work to collect or create materials themselves, which leads to the uncertain quality of materials, potential problems with copyright controversy, and a lack of comparability and generalization of experimental results.

Furthermore, most of these databases or study materials presented people/faces from Western countries, which may introduce confounding factors for Asian studies due to cultural differences (Boiger \& Mesquita, 2012). For example, it was found that Turkish and Indian participants (collectivist background) showed different responses to social exclusion compared to German and American participants (individualist background) (Pfundmair et al., 2015), possibly because people from collectivist backgrounds perceive social exclusion as less threatening so they are less affected by it than people from individualistic backgrounds (Pfundmair et al., 2018). The influence of culture is also reflected in the presence of "in-group advantage," i.e., individuals have stronger reactions to or better recognition of emotional information conveyed by individuals from the same race (Elfenbein \& Ambady, 2002; Brown et al., 2006). This in-group advantage has also been found in the emotional processing of social events such as social exclusion (He et al., 2021; Krill \& Platek, 2009). Considering the growing number of socialrelated studies from Asia, there is an urgent need for a standardized socioemotional image database.

This two-part research aims to create a standardized, open-access image database: the social inclusion/exclusion in young Asian adults (ISIEA) database, which displays three categories of social interaction contexts (social exclusion, social neutral, and social inclusion). Study 1 aims to establish and assess characteristics of ISIEA. Study 2 aims to explore the effect of image components (face and context) on the rating of the entire image.

\section{Study 1}

This study conducts a standardized assessment of images and provides ratings of valence, arousal, inclusion score, and vicarious feeling for each image. The ratings of valence 
(ranging from unpleasant to pleasant) and arousal (ranging from calm to excited) have been widely applied as basic dimensions of emotion in many social and affective studies (Lang et al., 1999; Kuppens et al., 2013). The inclusion score represents the participant's perceived intensity of social exclusion or social inclusion from the scenario in the images (Elliott et al., 2012). Vicarious feelings reflect vicarious affective experiences when participants imagine themselves as the highlighted person in the image (e.g., being rejected). While the ratings of valence, arousal, and inclusion were reported by participants with a relatively safe distance from the images, the rating of vicarious feeling reflects a more engaged affective experience in the social scenario. Previous research has found that participants experienced a stronger need threat when they took the perspective of the person in the image who was being socially excluded (Wesselmann et al., 2009). For all rating scales, we utilized semantic bipolar scales (a nine-point Likert scale). Although this scale is different from the Self-Assessment Manikin scale utilized by the IAPS, it has been shown to be highly correlated with the SAM scale (Bradley \& Lang, 1994) and has been adopted in many related studies (Kurdi et al., 2017; Marchewka et al., 2014; Teh et al., 2018). Since existing emotional image databases (e.g., IAPS; Lang et al., 1999) reveal that arousal and valence have an inverted U-shaped relationship, we thus hypothesized a similar quadratic relationship between arousal and valence/inclusion score/vicarious feelings. As an image database, the images' physical properties, including luminance, contrast, complexity, and color features, are also provided.

\section{Materials and methods}

\section{Participants}

A total of 63 healthy college students, including 37 females $(22.8 \pm 2.1$ years old, mean \pm standard deviation) and 26 males ( $23.2 \pm 1.8$ years old), were recruited from Shenzhen University to participate in the photographing as actors and actresses. For image ratings, we first recruited 100 healthy college students, including 50 females $(20.1 \pm 1.25$ years old) and 50 males $(20.7 \pm 2.09$ years old), from Shenzhen University to rate the images. Half a year after this initial sampling, an additional 50 healthy college students, comprising 21 females $(22.8 \pm 1.03$ years old $)$ and 29 males $(22.0 \pm 1.52$ years old), were recruited to add the sample size. These two samples of image ratings were combined for data analyses because of their homogeneity. To enhance the sample diversity, all participants consisted of undergraduate and graduate students from various majors, including natural science, social science, engineering, and medicine. The study was approved by the Ethics Committee of Shenzhen University. Informed consent was signed by the participants before the photography or the image rating task. All the actors and actresses authorized the use of their portraits for the ISIEA database after they clearly understood the informed consent form. After photographing or picture ratings, participants were paid $¥ 50$ as compensation.

\section{Materials}

A total of 164 images in the ISIEA were taken by our lab at Shenzhen University using a digital camera (Sony ILCE$7 \mathrm{M} 2$, Japan). To display various social situations on campus, we selected 104 outdoor scenes (playgrounds, school roads, lawns, etc.) and 60 indoor scenes (classrooms, libraries, offices, etc.) to enhance the diversity of the images' backgrounds. All images can be downloaded freely from the website https://doi.org/10.5281/zenodo.5496421.

As shown in Fig. 1a, there are three categories of images in the ISIEA. The "social exclusion" category $(N=60)$ depicts scenarios consisting of one rejectee with sad or upset facial and body expressions, and three or four rejecters talking and laughing together. The scenarios of the "social neutral" category $(N=53)$ are composed of two to five individuals with neutral expressions. No social interaction or communication existed in these images (i.e., individuals read books by themselves). The "social inclusion" category ( $N$ $=51$ ) of images includes social scenarios of friendly communication between three or four individuals.

\section{Physical properties of images}

Physical parameters, such as luminance, contrast, complexity, and color parameters, were measured utilizing the criteria in an existing affective image database (Marchewka et al., 2014). We utilized the Image Processing Toolbox in MATLAB (MathWorks, Natick, MA) to compute these parameters. Luminance is the average pixel value of a grayscale image, and contrast is the standard deviation across all pixels of the grayscale image (Bex \& Makous, 2002). The visual complexity of an image can be reflected by the JPG size and entropy. While the JPG size measures the overall complexity of an image (Donderi, 2006), entropy is a measure of randomness and reflects the texture of one image that was calculated using the MATLAB function entropy. $m$ in this study. For the color parameters, we calculated the pixel mean of each channel based on the CIE L*a*b* color space. Compared with the RGB color space, this color space is based on the opponent-process theory of color vision, so it is closer to the characteristics of the human visual system. The $\mathrm{L}^{*}$ channel represents the perceptual lightness ranging from 0 to 100; the a* channel represents the color spectrum range from green (negative values) to red (positive values), and the $b^{*}$ channel represents the color spectrum 
(a)
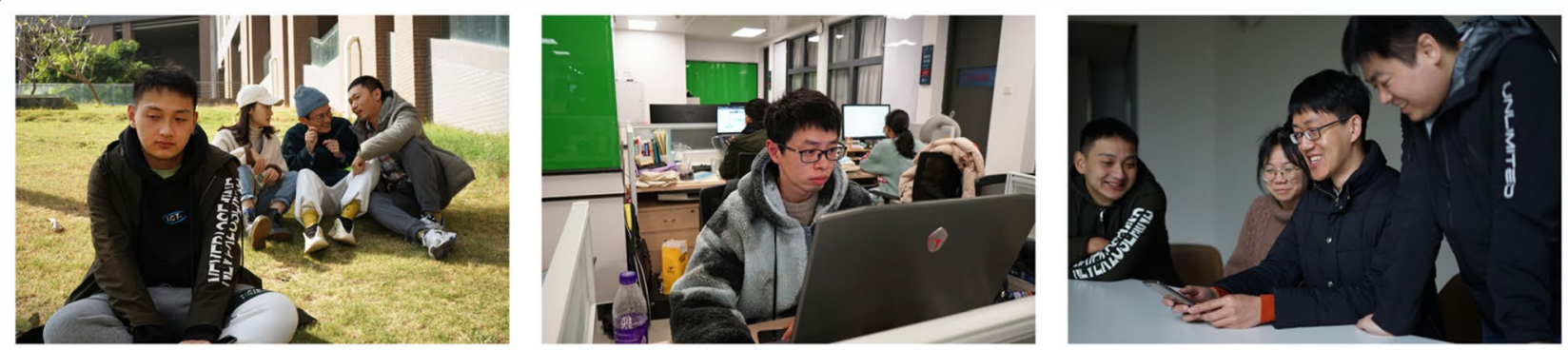

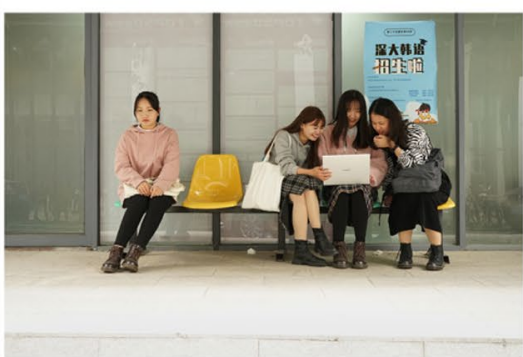

Social exclusion

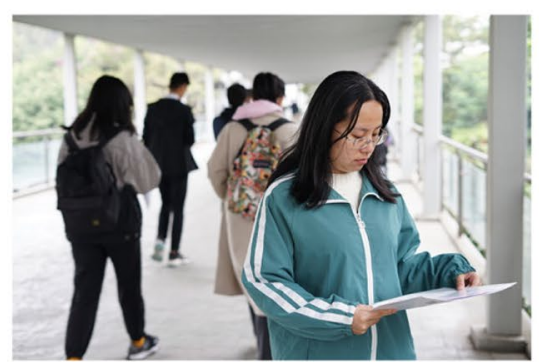

Social neutral

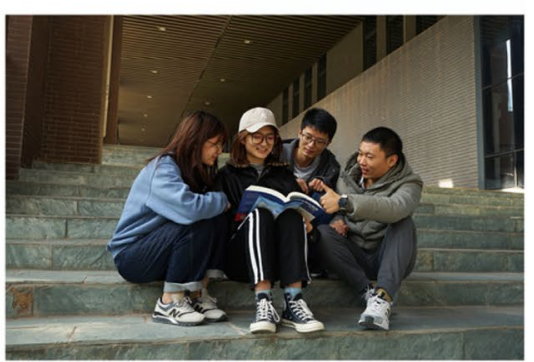

Social inclusion

(b)

\section{Block 1}

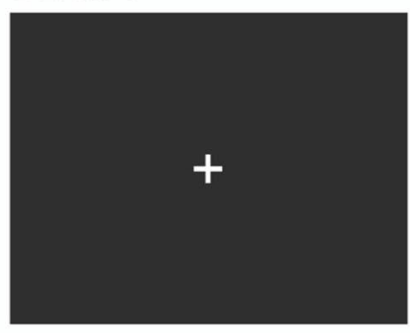

Fixation (0.5 s)

Block 2

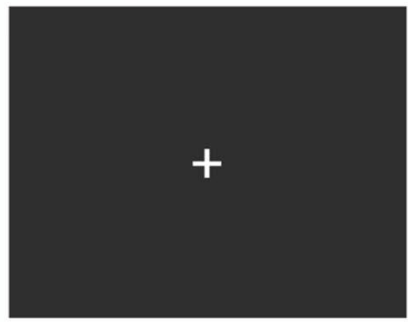

Fixation (0.5 s)

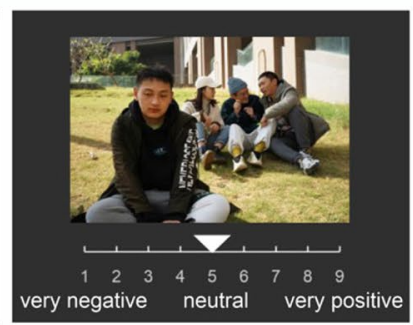

Valence (RT)

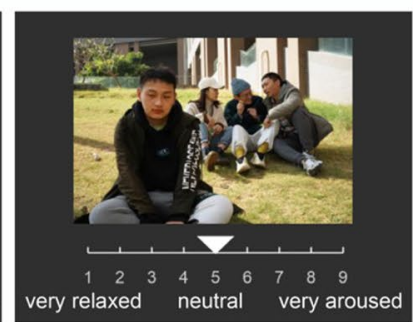

Arousal (RT)

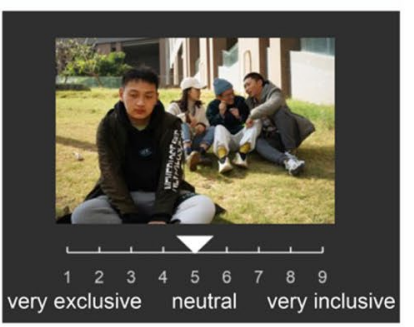

Inclusion score (RT)

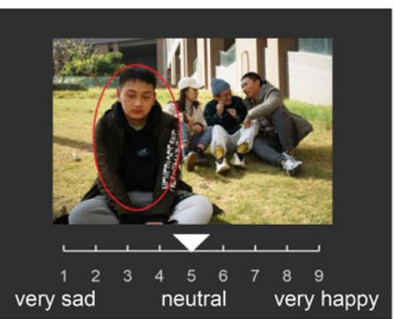

Vicarious feeling (RT)
Fig. 1 a Example pictures of social exclusion, social neutral, and social inclusion with female or male protagonists. b Trial design of the rating task. Each image remained on the screen until the participants finished their ratings ( $\mathrm{RT}=$ reaction time). The instructions/

range from blue (negative values) to yellow (positive values). The physical properties of each image are presented in Supplementary Material. All images were the same size, $1200 \times 800$ pixels (300 dpi). Imaging software Photoshop (Adobe Systems Incorporated, San Jose, CA, labels were displayed in Chinese during the task and translated to English for the sake of publication. The font color is white, and the background was gray $(\mathrm{RGB}=[50,50,50])$

USA) was used to adjust the color tone, brightness, and contrast of images to ensure that there was no difference in the measured physical characteristics across the three categories of images. 


\section{Picture rating}

Images were presented sequentially in a randomized order using PsychoPy software (version 3.1.0; Peirce et al., 2019) on an LCD screen with a viewing angle of $3.0^{\circ} \times 3.5^{\circ}$. Prior to rating, we utilized oral and written instructions to ensure that participants were clear about the meanings of the terminologies used in the task.

The rating procedure consisted of two blocks (Fig. 1b). In the first block, participants judged the valence (from $1=$ very negative, $5=$ neutral, to $9=$ very positive), then the arousal (from $1=$ very relaxed, $5=$ neutral, to $9=$ very aroused), and finally the degree of social inclusion (from $1=$ very exclusive, $5=$ neutral, to $9=$ very inclusive). We fixed the order of the three ratings for each image to avoid confusion and ensure that participants could rate valence, arousal, and inclusion level accurately (see also Teh et al., 2018). In the second block, all images appeared again, with a red circle highlighting one person in the image. Participants were instructed: "In this scenario, please think about how you would feel in a situation similar to that of the highlighted person in the image." Participants were then asked to report this vicarious affective feeling (from $1=$ very sad, 5 $=$ neutral, to $9=$ very happy) during image presentation. All ratings were reported by moving and clicking a bar on a horizontal scale using a computer mouse. Each image remained on the screen until participants finished their ratings. All 150 participants were presented twice with a complete set of 164 images in the two blocks. The entire rating procedure took approximately $40 \mathrm{~min}$. Participants had a break of 2-3 min at the middle of each block, and a break of 10 min between two blocks. After the image rating task, participants were asked to complete self-reported questionnaires, including the Liebowitz Social Anxiety Scale (LSAS, Liebowitz, 1987) and the Interpersonal Reactivity Index (IRI, Davis, 1980).

\section{Statistics}

Statistical analysis was performed using SPSS Statistics (version 20.0; IBM, Somers, NY). Descriptive data are presented as mean \pm standard deviation, unless otherwise stated. While we reported significant results in the main text for the sake of conciseness, a full report of statistical results could be found in Supplementary Material.

The ratings of valence, arousal, inclusion scores, and vicarious feelings were statistically analyzed. One-way ANOVAs were performed separately on each rating scale to compare the differences across the three categories of images. The Scheffe method was used to conduct a post hoc test (due to the unequal number of images across categories). Cronbach's $\alpha$ coefficient was used to measure the reliability of the image ratings across participants. Subsequently, the Pearson correlations between the ratings of valence, inclusion scores, and vicarious feelings were calculated. Owing to the quadratic relationship between arousal and valence, which has been widely reported in affective studies, we examined this relationship by fitting a quadratic regression. In addition, mixed design ANOVAs were performed to explore the time effect (details in Supplementary Material).

To explore the gender effect, $3 \times 2$ mixed design ANOVAs were performed separately for each rating scale, with the context category as the within-subject factor and gender as the between-subject factor (details in Supplementary Material). The potential influences of social anxiety and empathy of image raters were explored by conducting Pearson correlations between questionnaire scores and image ratings; multiple comparisons were corrected using the FDR method.

\section{Results}

\section{Ratings of valence, arousal, inclusion score, and vicarious feeling}

Ratings for each image are provided in Supplementary Material. The descriptive characteristics of the three categories are presented in Table 1. We also illustrate the rating distributions of the three categories of images in Fig. 2. The Kolmogorov-Smirnov test shows that the distributions of these ratings for the three categories of images conform to the normal distribution (all $p \geq .320$, see Table $\mathrm{S} 1$ for exact statistics).

One-way ANOVAs showed that the four ratings differed across the image categories (Fig. 3). For the valence of

Table 1 Descriptive statistics of picture ratings in the three categories of images

\begin{tabular}{|c|c|c|c|c|c|c|}
\hline \multirow[t]{2}{*}{ Rating } & \multicolumn{2}{|c|}{ Exclusion $(N=60)$} & \multicolumn{2}{|c|}{ Neutral $(N=53)$} & \multicolumn{2}{|c|}{ Inclusion $(N=51)$} \\
\hline & Mean \pm SD & Range & Mean \pm SD & Range & Mean \pm SD & Range \\
\hline Valance & $3.428 \pm 0.469$ & $2.490-4.650$ & $5.108 \pm 0.290$ & $4.420-5.680$ & $7.064 \pm 0.201$ & $6.590-7.440$ \\
\hline Arousal & $4.590 \pm 0.194$ & $4.100-5.110$ & $4.224 \pm 0.162$ & $3.940-4.720$ & $6.269 \pm 0.242$ & $5.720-6.630$ \\
\hline Inclusion score & $3.312 \pm 0.500$ & $2.200-4.470$ & $5.335 \pm 0.257$ & $4.650-5.810$ & $7.395 \pm 0.226$ & $6.850-7.720$ \\
\hline Vicarious feeling & $3.214 \pm 0.353$ & $2.270-4.070$ & $4.999 \pm 0.263$ & $4.070-5.590$ & $6.968 \pm 0.293$ & $6.390-7.540$ \\
\hline
\end{tabular}


(a)

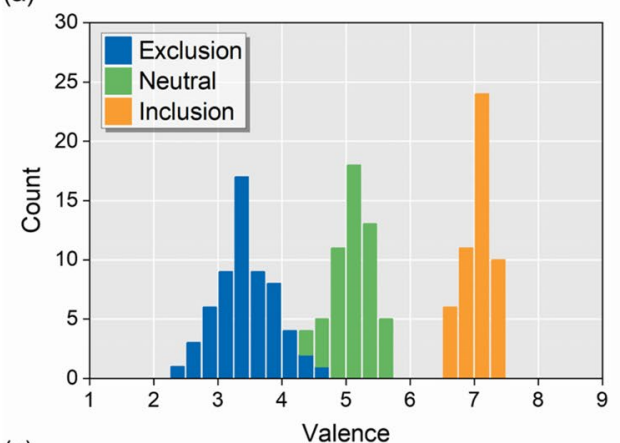

(c)

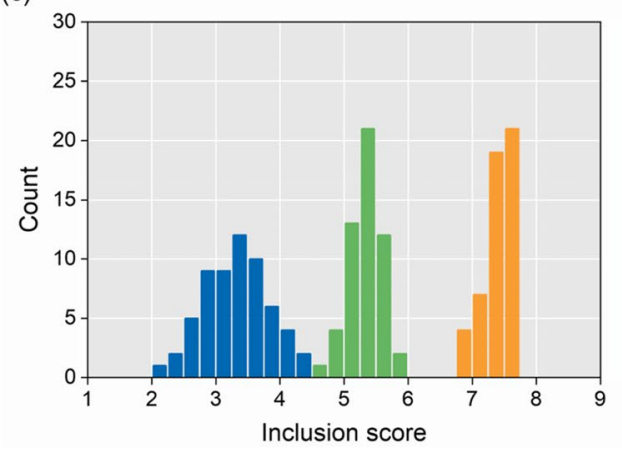

(b)

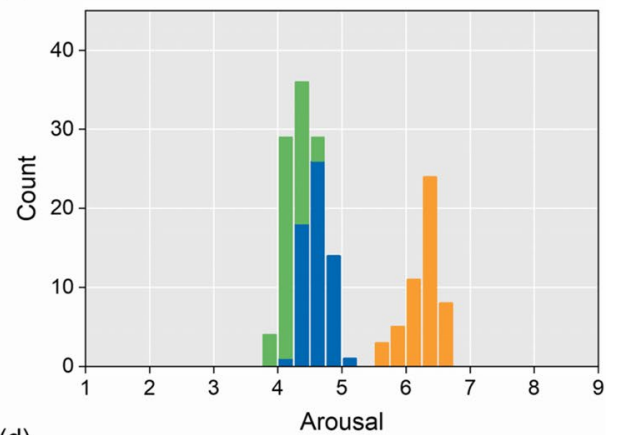

(d)

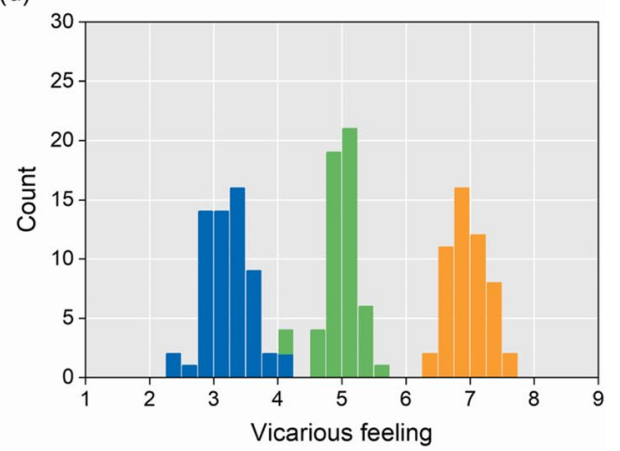

Fig. 2 Distribution of image ratings for a valence, $\mathbf{b}$ arousal, $\mathbf{c}$ inclusion score, and $\mathbf{d}$ vicarious feeling. The colors correspond to different image categories

images, $F(2,161)=1516.2, p<.001, \eta_{\mathrm{p}}^{2}=.950$, participants rated social inclusion images as the most positive, followed by social neutral images, and social exclusion images were rated as the most negative (pairwise $p$ s $<$ $.001)$. For the arousal rating, $F(2,161)=1537.9, p<.001$, $\eta_{\mathrm{p}}^{2}=.950$, participants reported the highest arousal for social inclusion images, followed by social exclusion images, and social neutral images were rated as having the lowest arousal (pairwise $p$ s $<.001$ ). For the inclusion score, $F(2,161)=1787.5, p<.001, \eta_{\mathrm{p}}^{2}=.957$, social exclusion images were rated as having the lowest scores (indicating a high level of social exclusion), and social inclusion images were rated as having the highest scores, with social neutral images having median scores indicating neither exclusive nor inclusive (pairwise $p \mathrm{~s}<.001$ ). For the vicarious feeling, $F(2,161)=2052.0, p<.001, \eta_{\mathrm{p}}^{2}=$ .962 , participants reported the most positive feelings for social inclusion images, followed by social neutral images, and social exclusion images were associated with the most negative feelings (pairwise $p s<.001$ ).

We also conducted a reliability analysis for these four ratings to examine the consistency across participants. The results showed excellent internal consistency for all ratings (Table 2).

\section{Relationships between picture ratings}

Correlation analysis showed a strong positive correlation between valence and inclusion score $(r=.998)$, between valence and vicarious feeling $(r=.988)$, and between inclusion score and vicarious feeling $(r=.988, p \mathrm{~s}<.001$; Fig. 4, panels a, c, e).

For the relationship between arousal and the other three ratings, quadratic regressions were performed (Table S2), and significant results were observed between arousal and valence $\left(R^{2}=.936\right)$, inclusion score $\left(R^{2}=.934\right)$, and vicarious feeling $\left(R^{2}=.923\right.$, all $p \mathrm{~s}<.001$; Fig. 4 , panels $\mathrm{b}$, d, f). Generally, images with a high positive and negative valence or vicarious feelings, and images showing socially inclusive or exclusive scenarios were associated with high arousal scores.

\section{Effect of gender, social anxiety, and empathy on picture ratings}

The gender effect was examined utilizing two-way mixed design ANOVA (Table S4), which showed that neither the main effect of gender nor the interaction between gender and context was significant (all $p \geq .440$, details in Supplementary Material). These results suggested that gender has no effect on images' ratings. 
(a)

(c)
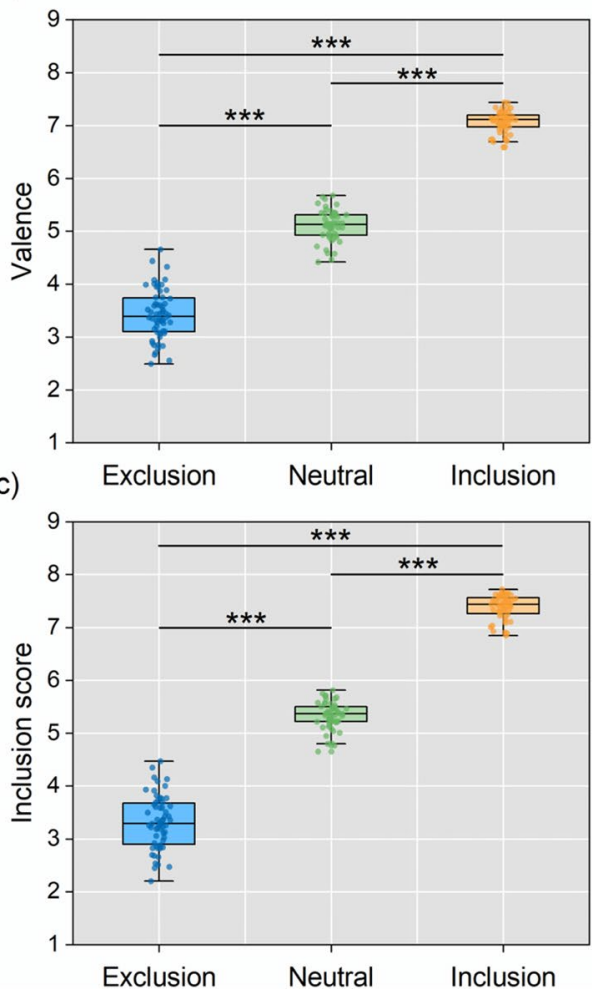

Fig. 3 Picture ratings for the three categories for $\mathbf{a}$ valence, $\mathbf{b}$ arousal, $\mathbf{c}$ inclusion, and $\mathbf{d}$ vicarious feeling. Each dot represents the mean rating of an image; boxes represent the 25 th and 75 th percentiles, and

Correlation analyses were performed between social anxiety/empathy and the four image ratings (Table S3). Results show that social anxiety was negatively correlated with valence $\left(r=-.327, p<.001, p_{\text {cor }}<.001\right)$, inclusion score $(r$ $\left.=-.193, p=.018, p_{c o r}=.043\right)$, and vicarious feelings $(r=$ $\left.-.324, p<.001, p_{c o r}<.001\right)$ of social neutral images, which indicated that people with higher social anxiety tended to have more negative feelings in socially neutral scenarios. Social anxiety was also negatively correlated with vicarious feelings $\left(r=-.199, p=.015, p_{c o r}=.039\right)$, indicating people with high social anxiety have more negative feelings when vicariously experiencing social exclusion scenarios. Empathy was positively correlated with valence $(r=.314$, $\left.p<.001, p_{\text {cor }}<.001\right)$, inclusion score $(r=.341, p<.001$, $\left.p_{c o r}<.001\right)$, and vicarious feeling $\left(r=.287, p<.001, p_{c o r}\right.$

Table 2 Reliability (Cronbach's alpha) of picture ratings

\begin{tabular}{llll}
\hline Rating & Exclusion & Neutral & Inclusion \\
\hline Valance & 0.976 & 0.935 & 0.973 \\
Arousal & 0.984 & 0.984 & 0.987 \\
Inclusion score & 0.977 & 0.978 & 0.976 \\
Vicarious feeling & 0.978 & 0.906 & 0.961 \\
\hline
\end{tabular}

(b)

(d)
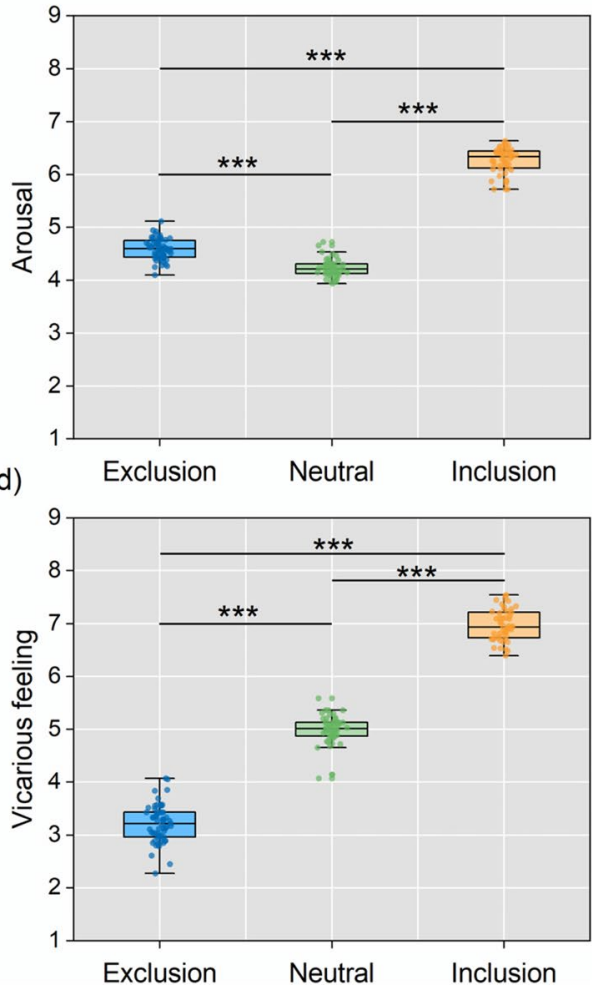

the whiskers represent upper and lower values within $1.5 \times$ interquartile range. Pairwise comparison: $* * * p<0.001$

$=.002$ ) of social inclusion images, suggesting people with higher levels of empathy rate social inclusion images as more positive and more inclusive. Empathy was also negatively correlated with valence $\left(r=-.255, p=.002, p_{c o r}=\right.$ $.005)$, inclusion score $\left(r=-.225, p=.006, p_{c o r}=.017\right)$, and vicarious feeling $\left(r=-.269, p<.001, p_{c o r}=.003\right)$ of social exclusion images, indicating people with higher levels of empathy rate social exclusion images as more negative and more exclusive.

\section{Study 2}

This study investigated whether image ratings were independently influenced by facial expressions or social contexts of images. Specifically, every image was segmented into face and context, forming two new sets of images (Fig. 5). Ratings for these two image sets were collected to examine the impact of facial expression and social context. 
(a)

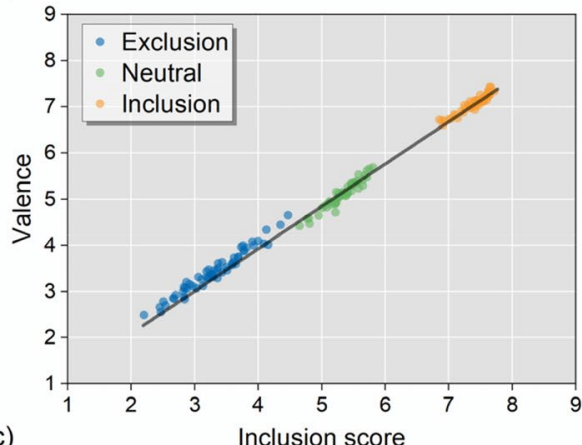

(c)

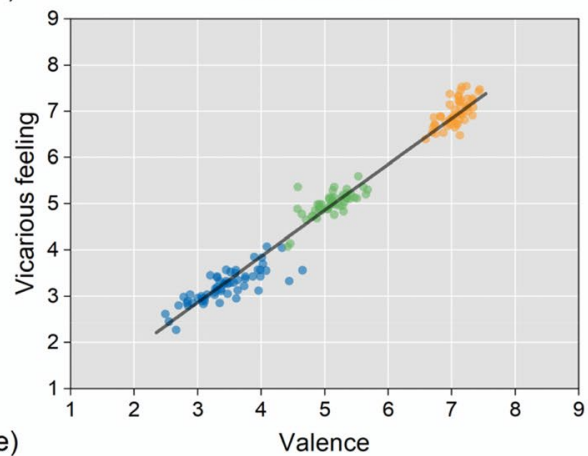

(e)

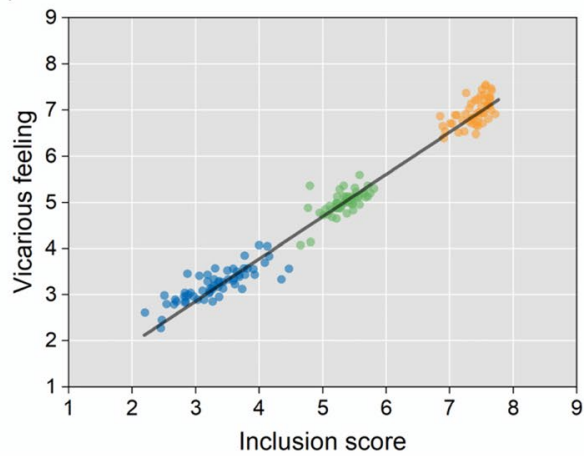

Fig. 4 Relationships between a valence and inclusion score, $\mathbf{b}$ arousal and valence, $\mathbf{c}$ vicarious feeling and valence, $\mathbf{d}$ arousal and inclusion score, $\mathbf{e}$ vicarious feeling and inclusion score, and $\mathbf{f}$ arousal and vicar-

\section{Materials and methods}

\section{Participants}

Another 50 healthy college students, comprising 24 females $(21.1 \pm 1.4$ years old $)$ and 26 males $(21.8 \pm 1.9$ years old), were recruited from Shenzhen University. The participants samples of the two studies were homogeneous. Informed consent was signed before the image rating task. After image ratings, each participant was paid $¥ 50$ as compensation. (b)

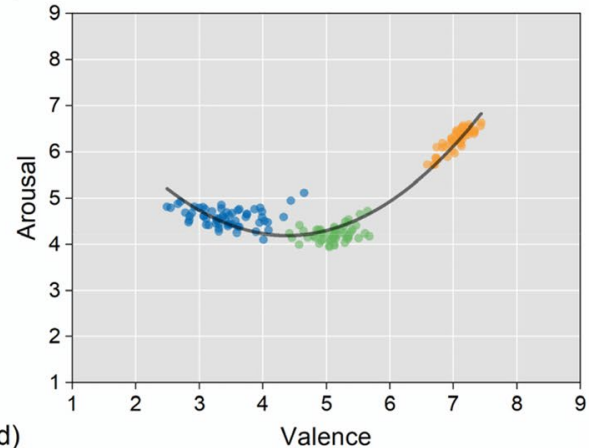

(d)
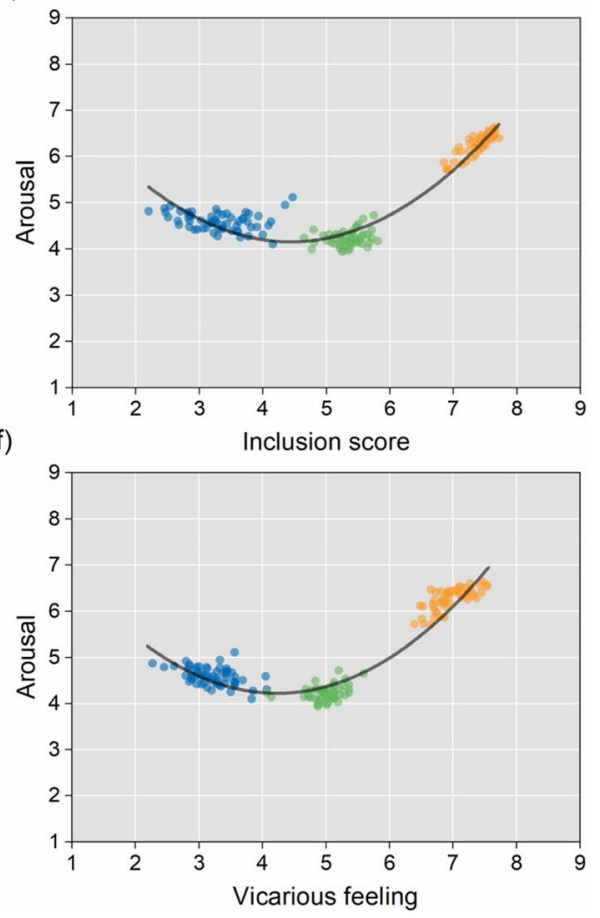

ious feeling. Each dot represents the mean rating of an image. Colors correspond to different image categories

\section{Materials}

For the face set, the face of the main character was extracted and placed on a gray background using Photoshop (Adobe Systems Incorporated, San Jose, CA, USA). For the context set, the face of the main character was masked by mosaic while the rest of the image was preserved as the context component. Both sets of images had the same size and resolution $(1200 \times 800$ pixels, $300 \mathrm{dpi})$.

\section{Picture rating}

The rating task was divided into two blocks, corresponding to the face and context image sets. The order of blocks was counterbalanced across subjects. In the face 
(a)

(b)

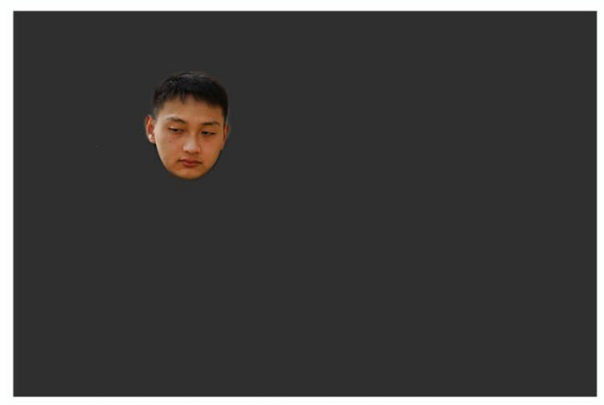

Face

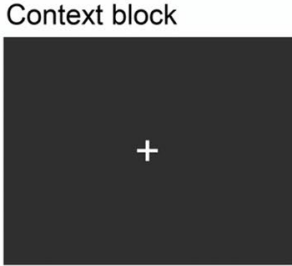

Fixation $(0.5 \mathrm{~s})$

Face block

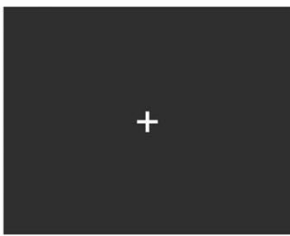

Fixation $(0.5 \mathrm{~s})$

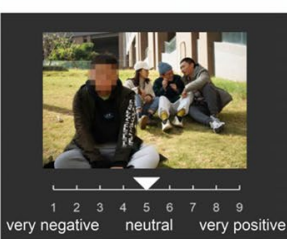

Valence (RT)

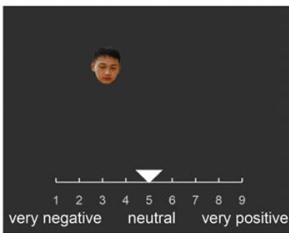

Valence (RT)

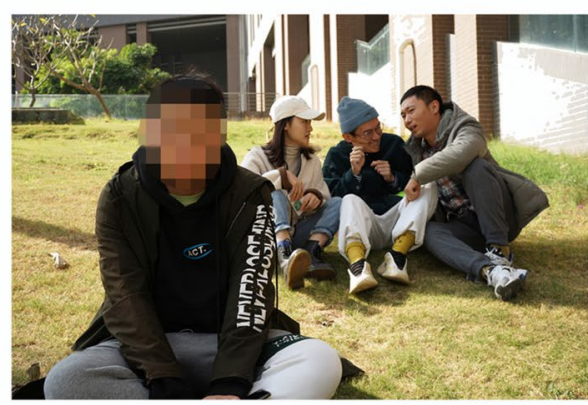

Context

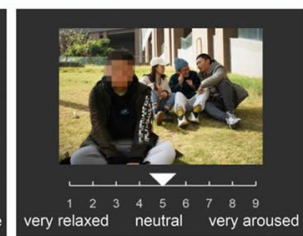

Arousal (RT)

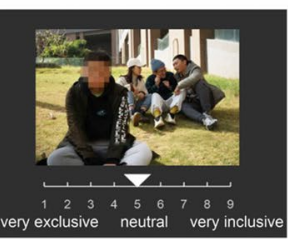

Inclusion score (RT)

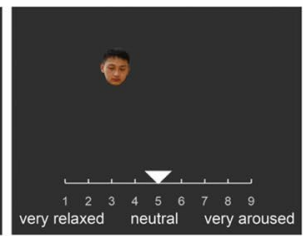

Arousal (RT)
Fig. 5 a Example pictures of face and context component. b The trial design of the rating task. Each image remained on the screen until the participants finished their ratings $(\mathrm{RT}=$ reaction time). The instruc-

block, participants judged the valence and arousal of each face image. In the context block, participants judged the valence, arousal, and inclusion levels of each context image. All the images were presented randomly with a fixed order of various ratings. The entire rating procedure took approximately $40 \mathrm{~min}$. The rest setting was consistent with Study 1.

\section{Statistics}

Statistical analysis was performed using SPSS Statistics (version 20.0; IBM, Somers, NY, USA). Regression analyses were performed to investigate the influence of face/context ratings on the ratings of the entire image (enter method). First, two multiple regression models were fitted to predict the valence of the entire image using valence ratings of face and context as predictors, and to predict the arousal of the entire image using arousal ratings of face and context as predictors. Second, two multiple regression models were fitted to predict the inclusion score of the entire image using tions/labels were displayed in Chinese during the task and translated to English for the sake of publication. The font color is white, and the background was gray $(\mathrm{RGB}=[50,50,50])$

valence ratings of face and context as predictors, and to predict the inclusion score of the entire image using valence ratings of face and inclusion score of the context as predictors.

\section{Results}

Regression analysis revealed that the ratings of both face and context images positively predicted the ratings of entire images (Table 3), indicating that the emotional attributes of face and context components independently affect the emotional attributes of the entire image. Descriptive statistics are reported in Table 4.

\section{Discussion}

Introducing the ISIEA database and its utilization in this study has shown promise. The ratings and physical parameters provided for these pictures in color (Supplementary Material) would be helpful for future image selection and control of confounding factors. 
Table 3 Summary of regression analyses $(N=163)$

\begin{tabular}{lllll}
\hline Model & $\beta$ & $\beta_{\text {standardized }}$ & $\mathrm{t}$ & $p$ \\
\hline
\end{tabular}

(a) Dependent variable: valence of entire image

$\mathrm{R}^{2}=.974, F(2,161)=3024, p<.001$

$\begin{array}{lllll}\text { Face valence } & 0.402 & 0.344 & 11.657 & <.001 \\ \text { Context valence } & 0.779 & 0.665 & 22.505 & <.001\end{array}$

(b) Dependent variable: arousal of entire image

$\mathrm{R}^{2}=.961, F(2,161)=1973, p<.001$

$\begin{array}{lllll}\text { Face arousal } & 0.364 & 0.402 & 13.585 & <.001 \\ \text { Context arousal } & 0.632 & 0.616 & 20.808 & <.001\end{array}$

(c) Dependent variable: inclusion of entire image

$\mathrm{R}^{2}=.969, F(1,161)=2496, p<.001$

$\begin{array}{lllll}\text { Face valence } & 0.369 & 0.283 & 8.713<.001 \\ \text { Context valence } & 0.946 & 0.722 & 22.252 & <.001\end{array}$

(d) Dependent variable: inclusion of entire image

$\mathrm{R}^{2}=.967, F(1,161)=3933, p<.001$

\begin{tabular}{lllll} 
Face valence & 0.337 & 0.258 & 10.087 & $<.001$ \\
Context inclusion scores & 0.781 & 0.751 & 29.345 & $<.001$ \\
\hline
\end{tabular}

Category comparisons using ANOVA demonstrated significant differences in image ratings across socially inclusive, neutral, and exclusive images. Social exclusion images were rated as the most negative (lowest valence) and the most socially exclusive (lowest inclusion score), and evoked the most negative feelings (lowest vicarious feeling). The social inclusion images were rated as the most positive (highest valence) and the most socially inclusive (highest inclusion score), and evoked the most positive feelings (highest vicarious feeling). The social neutral images were rated as neither negative nor positive (medium valence), neither exclusive nor inclusive (medium inclusion score), and evoked neutral feelings (medium vicarious feeling). Regarding the arousal rating, the highest scores were obtained for social inclusion images, possibly due to more explicit social interactions with intense facial expressions (i.e., laughter) or obvious body language (i.e., waving hand). Meanwhile, social exclusion images had stronger arousal than social neutral images, although the difference between these two categories was smaller due to the relatively low arousal of social exclusion images (individuals usually displayed sad, upset, or calm facial and body expressions). The high Cronbach's $\alpha$ coefficients for the four ratings indicated high agreement among participants on the emotional experience and interpretation of social content in each context category.

Regarding the relationship between these picture ratings, the strong positive correlation between valence, inclusion score, and vicarious feeling suggest that the valence and vicarious affective feelings are highly related to the level of social exclusion/inclusion that participants perceived from the scenarios. The relationship between valence and arousal was quadratic. This typical boomerang shape between valence and arousal has been widely reported in many databases (Kurdi et al., 2017; Lang et al., 1999; Marchewka et al., 2014). Likewise, a quadratic relationship was found between arousal and inclusion score/vicarious feeling, indicating that images with high social inclusion and exclusion levels and images perceived as having strong positive and negative vicarious emotions are more arousing than images with a neutral social context.

We also explored the potential influential effects on image ratings. First, individuals' social anxiety magnified the negative feelings they experienced from socially neutral and exclusion images. This is consistent with previous studies reporting that socially anxious individuals tend to negatively interpret ambiguous social information (Mobini et al., 2013; Peschard \& Philippot, 2017), and that they are sensitive and show strong negative emotional responses to various events that cause social distress (Auyeung \& Alden, 2016; Goldin et al., 2009; Harb et al., 2002). Second, we found that a high capacity for empathy enhanced participants' emotional experience toward exclusion and inclusion images. The ability of empathy is the cornerstone of social function, enabling us to understand others' mental and affective states (Singer \& Lamm, 2009). Numerous studies have shown that observed and imagined experiences of social exclusion shared similar emotional experiences and neural responses (Eisenberger, 2012; Giesen \& Echterhoff, 2018; Meyer et al., 2013; Wesselmann et al., 2009). For instance, Masten et al. (2011) found that trait empathy is positively correlated with the neural activity of empathy-related brain regions when participants experienced social exclusion displayed in images, suggesting that the emotional experience induced by exclusion images is significantly influenced by empathic ability. In general, these findings suggest that researchers should

Table 4 Descriptive statistics of picture ratings for face, context, and the entire image

\begin{tabular}{|c|c|c|c|c|c|c|}
\hline \multirow[t]{2}{*}{ Component } & \multicolumn{2}{|l|}{ Exclusion } & \multicolumn{2}{|l|}{ Neutral } & \multicolumn{2}{|l|}{ Inclusion } \\
\hline & Valence & Arousal & Valence & Arousal & Valence & Arousal \\
\hline Face & $3.85 \pm 0.49$ & $3.96 \pm 0.26$ & $4.80 \pm 0.55$ & $4.04 \pm 0.38$ & $6.79 \pm 0.39$ & $5.97 \pm 0.48$ \\
\hline Context & $3.82 \pm 0.59$ & $4.75 \pm 0.23$ & $5.21 \pm 0.28$ & $4.18 \pm 0.28$ & $6.83 \pm 0.33$ & $6.18 \pm 0.37$ \\
\hline Entire & $3.43 \pm 0.47$ & $4.59 \pm 0.19$ & $5.11 \pm 0.29$ & $4.22 \pm 0.16$ & $7.06 \pm 0.20$ & $6.27 \pm 0.24$ \\
\hline
\end{tabular}


consider individual differences when employing our image database in their studies.

In Study 2, we examined the role of facial expression and social context in the emotional rating of ISIEA. While isolated facial expressions usually transfer clear basic emotions, their interpretation becomes more complicated in socially interactive contexts. For example, facial expressions of laughter and frustration may coexist in scenes of social exclusion. In these cases, contextual information that helps us accurately perceive and interpret others' emotions is critical (Olsson \& Ochsner, 2008). Our results demonstrated that the emotional attributes of both facial expression and social context contribute to the ratings of an entire image, suggesting that social context is essential for delivering inclusion/exclusion information.

Furthermore, we suggest considering the following aspects when using images from this database. First, the social exclusion images were produced according to a narrow definition of social exclusion or ostracism (Williams, 2007) that did not include images displaying overt abuse, finger-pointing reproach, or other strong negative facial expressions (e.g., crying and fear). This is also the reason for the lack of high-arousal images in the social exclusion category. Second, while this database contains images of and is rated by young adults, people of different ages may have different interpretations, emotional experiences, and neural responses to context-based social images. For instance, a relevant meta-analysis study found that adults mainly activated the ventrolateral prefrontal cortex, while adolescents mainly activated the medial prefrontal cortex during social exclusion (Vijayakumar et al., 2017). Third, while the fixed order for rating valence, arousal, and inclusion avoided confusion during the task, this manipulation introduced a potential order contamination to the image ratings (Kawai et al., 2021; Kurdi et al., 2017). Fourth, our rating task lasted approximately 40 min, which introduced a slight habituation (or fatigue) effect on the image ratings (Table S5 and Fig. S1). Fortunately, images were presented in a random order (i.e., the order differed between participants), which counterbalanced the habituation effect across various images.

Despite these considerations, possible applications of ISIEA are broad. First, it can be applied to studies investigating social and emotional processing in the context of social exclusion/inclusion. These studies include attention allocation, memory, social reward/punishment anticipation and evaluation, and emotion regulation of socially evoked feelings. Second, besides investigating social exclusion/inclusion per se, these context-based images can be utilized as affective priming stimuli to induce socially positive or negative emotions, thus facilitating an investigation of follow-up cognitive processes (e.g., decision-making and learning), social behaviors (e.g., prosocial and vengeance trends), and individual characteristics (e.g., self-esteem and state anxiety) influenced by social emotions. Third, the application of the database in clinical research will be beneficial for examining social dysfunctions in clinical populations, such as autism spectrum disorder and alexithymia (Happé et al., 2017).

\section{Conclusions}

The ISIEA database we developed consists of 164 photographs, divided into three social context categories (social exclusion, social neutral, and social inclusion), and is available for free download from https://doi.org/10.5281/zenodo. 5496421 . By referring to the standardized ratings provided, researchers can choose the appropriate images and flexibly conduct experimental control. We hope that ISIEA will facilitate standardization and comparability across related studies as a valid resource and contribute to promoting the development of socio-emotional studies.

Supplementary Information The online version contains supplementary material available at https://doi.org/10.3758/s13428-021-01736-w.

Data availability The data and code of this study would be available upon reasonable request and with approvals of School of Psychology, Shenzhen University. More information on making this request can be obtained from the corresponding author, Prof. Dandan Zhang (zhangdd05@gmail.com).

Author Contribution DZ and $\mathrm{ZZ}$ conceived the study and wrote the manuscript. ZZ, SL, LM, and WC performed the experiment and collected data. ZZ analyzed the data.

Funding This research was supported by the National Natural Science Foundation of China (31970980; 31871115), and Shenzhen-Hong Kong Institute of Brain Science (2021SHIBS0003).

\section{Declarations}

Conflicts of interest Authors declare no conflicts of interest in relation to the subject of this study.

Open Access This article is licensed under a Creative Commons Attribution 4.0 International License, which permits use, sharing, adaptation, distribution and reproduction in any medium or format, as long as you give appropriate credit to the original author(s) and the source, provide a link to the Creative Commons licence, and indicate if changes were made. The images or other third party material in this article are included in the article's Creative Commons licence, unless indicated otherwise in a credit line to the material. If material is not included in the article's Creative Commons licence and your intended use is not permitted by statutory regulation or exceeds the permitted use, you will need to obtain permission directly from the copyright holder. To view a copy of this licence, visit http://creativecommons.org/licenses/by/4.0/. 


\section{References}

Auyeung, K. W., \& Alden, L. E. (2016). Social anxiety and empathy for social pain. Cognitive Therapy and Research, 40(1), 38-45. https://doi.org/10.1007/s10608-015-9718-0

Barrett L. F. (2006). Are emotions natural kinds? Perspectives on Psychological Science: A Journal of the Association for Psychological Science, 1(1), 28-58. https://doi.org/10.1111/j.1745-6916. 2006.00003.x

Baumeister, R. F., \& Leary, M. R. (1995). The need to belong: desire for interpersonal attachments as a fundamental human motivation. Psychological Bulletin, 117(3), 497-529.

Bex, P. J., \& Makous, W. (2002). Spatial frequency, phase, and the contrast of natural images. Journal of the Optical Society of America. A, Optics, Image Science, and Vision, 19(6), 1096-1106. https:// doi.org/10.1364/josaa.19.001096

Boiger, M., \& Mesquita, B. (2012). The construction of emotion in interactions, relationships, and cultures. Emotion Review, 4(3), 221-229. https://doi.org/10.1177/1754073912439765

Bradley, M. M., \& Lang, P. J. (1994). Measuring emotion: the SelfAssessment Manikin and the Semantic Differential. Journal of Behavior Therapy and Experimental Psychiatry, 25(1), 49-59. https://doi.org/10.1016/0005-7916(94)90063-9

Brown, L. M., Bradley, M. M., \& Lang, P. J. (2006). Affective reactions to pictures of ingroup and outgroup members. Biological Psychology, 71(3), 303-311. https://doi.org/10.1016/j.biopsycho. 2005.06.003

Dalgleish T. (2004). The emotional brain. Nature Reviews. Neuroscience, 5(7), 583-589. https://doi.org/10.1038/nrn1432

Dan-Glauser, E. S., \& Scherer, K. R. (2011). The Geneva affective picture database (GAPED): a new 730-picture database focusing on valence and normative significance. Behavior Research Methods, 43(2), 468-477. https://doi.org/10.3758/s13428-011-0064-1

Davis, M. H. 1980. A multidimensional approach to individual differences in empathy. JSAS Catalog of Selected Documents in Psychology, 10, 85 .

Donderi, D. C. (2006). Visual complexity: A review. Psychological Bulletin, 132, 73-97. https://doi.org/10.1037/0033-2909.132.1.73

Eisenberger N. I. (2012). The pain of social disconnection: examining the shared neural underpinnings of physical and social pain. Nature reviews. Neuroscience, 13(6), 421-434. https://doi.org/10. 1038/nrn3231

Eisenberger N. I. (2013). Social ties and health: a social neuroscience perspective. Current Opinion in Neurobiology, 23(3), 407-413. https://doi.org/10.1016/j.conb.2013.01.006

Eisenberger N. I. (2015). Social pain and the brain: controversies, questions, and where to go from here. Annual Review of Psychology, 66, 601-629. https://doi.org/10.1146/annur ev-psych-010213-115146

Eisenberger, N. I., Lieberman, M. D., \& Williams, K. D. (2003). Does rejection hurt? An FMRI study of social exclusion. Science (New York, N.Y.), 302(5643), 290-292. https://doi.org/10.1126/science. 1089134

Eisenberger, N. I., Master, S. L., Inagaki, T. K., Taylor, S. E., Shirinyan, D., Lieberman, M. D., \& Naliboff, B. D. (2011). Attachment figures activate a safety signal-related neural region and reduce pain experience. Proceedings of the National Academy of Sciences of the United States of America, 108(28), 11721-11726. https://doi. org/10.1073/pnas.1108239108

Ekman P. (1992). Are there basic emotions? Psychological Review, 99(3), 550-553. https://doi.org/10.1037/0033-295x.99.3.550

Elfenbein, H. A., \& Ambady, N. (2002). On the universality and cultural specificity of emotion recognition: a meta-analysis. Psychological Bulletin, 128(2), 203-235. https://doi.org/10.1037/00332909.128.2.203
Elliott, R., Lythe, K., Lee, R., McKie, S., Juhasz, G., Thomas, E. J., Downey, D., Deakin, J. F., \& Anderson, I. M. (2012). Reduced medial prefrontal responses to social interaction images in remitted depression. Archives of General Psychiatry, 69(1), 37-45. https://doi.org/10.1001/archgenpsychiatry.2011.139

Giesen, A., \& Echterhoff, G. (2018). Do I really feel your pain? Comparing the effects of observed and personal ostracism. Personality and Social Psychology Bulletin, 44(4), 550-561. https://doi.org/ 10.1177/0146167217744524

Goldin, P. R., Manber, T., Hakimi, S., Canli, T., \& Gross, J. J. (2009). Neural bases of social anxiety disorder: Emotional reactivity and cognitive regulation during social and physical threat. Archives of General Psychiatry, 66(2), 170. https://doi.org/10.1001/archg enpsychiatry.2008.525

Happé, F., Cook, J. L., \& Bird, G. (2017). The Structure of Social Cognition: In(ter)dependence of Sociocognitive Processes. Annual Review of Psychology, 68, 243-267. https://doi.org/10.1146/annur ev-psych-010416-044046

Harb, G. C., Heimberg, R. G., Fresco, D. M., Schneier, F. R., \& Liebowitz, M. R. (2002). The psychometric properties of the Interpersonal Sensitivity Measure in social anxiety disorder. Behaviour Research and Therapy, 40(8), 961-979. https://doi. org/10.1016/S0005-7967(01)00125-5

He, Z., Lin, Y., Xia, L., Liu, Z., Zhang, D., \& Elliott, R. (2018). Critical role of the right VLPFC in emotional regulation of social exclusion: A tDCS study. Social Cognitive and Affective Neuroscience, 13(4), 357-366. https://doi.org/10.1093/scan/nsy026

He, Z., Liu, Z., Zhao, J., Elliott, R., \& Zhang, D. (2020a). Improving emotion regulation of social exclusion in depression-prone individuals: a tDCS study targeting right VLPFC. Psychological Medicine, 50(16), 2768-2779. https://doi.org/10.1017/S0033 291719002915

He, Z., Zhao, J., Shen, J., Muhlert, N., Elliott, R., \& Zhang, D. (2020b). The right VLPFC and downregulation of social pain: A TMS study. Human Brain Mapping, 41(5), 1362-1371. https://doi.org/ $10.1002 / \mathrm{hbm} .24881$

He, Z., Muhlert, N., \& Elliott, R. (2021). Emotion regulation of social exclusion: A cross-cultural study. Humanities and Social Sciences Communications, 8(1), 1-7. https://doi.org/10.1057/ s41599-021-00857-z

Kawai, C., Lukács, G., \& Ansorge, U. (2021). A new type of pictorial database: The Bicolor Affective Silhouettes and Shapes (BASS). Behavior Research Methods, https://doi.org/10.3758/s13428-02101569-7. Advance online publication.

Keysers, C., \& Gazzola, V. (2007). Integrating simulation and theory of mind: from self to social cognition. Trends in Cognitive Sciences, 11(5), 194-196. https://doi.org/10.1016/j.tics.2007.02.002

Kiecolt-Glaser, J. K., Gouin, J. P., \& Hantsoo, L. (2010). Close relationships, inflammation, and health. Neuroscience and Biobehavioral Reviews, 35(1), 33-38. https://doi.org/10.1016/j.neubiorev. 2009.09.003

Kok, B. E., Coffey, K. A., Cohn, M. A., Catalino, L. I., Vacharkulksemsuk, T., Algoe, S. B., Brantley, M., \& Fredrickson, B. L. (2013). How positive emotions build physical health: perceived positive social connections account for the upward spiral between positive emotions and vagal tone. Psychological Science, 24(7), 11231132. https://doi.org/10.1177/0956797612470827

Krill, A., \& Platek, S. (2009). In-group and out-group membership mediates anterior cingulate activation to social exclusion. Frontiers in Evolutionary Neuroscience, 1, 1. https://doi.org/10.3389/ neuro.18.001.2009

Kross, E., Egner, T., Ochsner, K., Hirsch, J., \& Downey, G. (2007). Neural dynamics of rejection sensitivity. Journal of Cognitive Neuroscience, 19(6), 945-956. https://doi.org/10.1162/jocn.2007. 19.6.945 
Kuppens, P., Tuerlinckx, F., Russell, J. A., \& Barrett, L. F. (2013). The relation between valence and arousal in subjective experience. Psychological Bulletin, 139(4), 917-940. https://doi.org/10.1037/ a0030811

Kurdi, B., Lozano, S., \& Banaji, M. R. (2017). Introducing the Open Affective Standardized Image Set (OASIS). Behavior Research Methods, 49(2), 457-470. https://doi.org/10.3758/ s13428-016-0715-3

Lang, P. J., Bradley, M. M., \& Cuthbert, B. N. (1999). International Affective Picture System (IAPS). Instruction Manual and Affective Ratings (Technical Report No. A-4). University of Florida, Center for Research in Psychophysiology.

Liebowitz M. R. (1987). Social phobia. Modern Problems of Pharmacopsychiatry, 22, 141-173. https://doi.org/10.1159/000414022

Ma, D. S., Correll, J., \& Wittenbrink, B. (2015). The Chicago face database: A free stimulus set of faces and norming data. Behavior Research Methods, 47(4), 1122-1135. https://doi.org/10.3758/ s13428-014-0532-5

Marchewka, A., Zurawski, Ł., Jednoróg, K., \& Grabowska, A. (2014). The Nencki Affective Picture System (NAPS): introduction to a novel, standardized, wide-range, high-quality, realistic picture database. Behavior Research Methods, 46(2), 596-610. https:// doi.org/10.3758/s13428-013-0379-1

Masten, C. L., Morelli, S. A., \& Eisenberger, N. I. (2011). An fMRI investigation of empathy for 'social pain' and subsequent prosocial behavior. NeuroImage, 55(1), 381-388. https://doi.org/10. 1016/j.neuroimage.2010.11.060

Meyer, M. L., Masten, C. L., Ma, Y., Wang, C., Shi, Z., Eisenberger, N. I., \& Han, S. (2013). Empathy for the social suffering of friends and strangers recruits distinct patterns of brain activation. Social Cognitive and Affective Neuroscience, 8(4), 446-454. https://doi. org/10.1093/scan/nss019

Mobini, S., Reynolds, S., \& MacKintosh, B. (2013). Clinical implications of cognitive bias modification for interpretative biases in social anxiety: An integrative literature review. Cognitive Therapy and Research, 37(1), 173-182. https://doi.org/10.1007/ s10608-012-9445-8

Olsson, A., \& Ochsner, K. N. (2008). The role of social cognition in emotion. Trends in Cognitive Sciences, 12(2), 65-71. https://doi. org/10.1016/j.tics.2007.11.010

Peirce, J., Gray, J. R., Simpson, S., MacAskill, M., Höchenberger, R., Sogo, H., Kastman, E., \& Lindeløv, J. K. (2019). PsychoPy2: Experiments in behavior made easy. Behavior Research Methods, 51(1), 195-203. https://doi.org/10.3758/s13428-018-01193-y

Peschard, V., \& Philippot, P. (2017). Overestimation of threat from neutral faces and voices in social anxiety. Journal of Behavior Therapy and Experimental Psychiatry, 57, 206-211. https://doi. org/10.1016/j.jbtep.2017.06.003

Pfundmair, M., Graupmann, V., Frey, D., \& Aydin, N. (2015). The Different Behavioral Intentions of Collectivists and Individualists in Response to Social Exclusion. Personality and Social Psychology
Bulletin, 41(3), 363-378. https://doi.org/10.1177/0146167214 566186

Pfundmair, M., Graupmann, V., Frey, D., \& Aydin, N. (2018). Interkulturelle Unterschiede im Erleben und Verhalten nach sozialer Exkludierung. Psychologische Rundschau, 69(2), 95-103. https:// doi.org/10.1026/0033-3042/a000343

Premkumar, P., Ettinger, U., Inchley-Mort, S., Sumich, A., Williams, S. C., Kuipers, E., \& Kumari, V. (2012). Neural processing of social rejection: the role of schizotypal personality traits. Human Brain Mapping, 33(3), 695-706. https://doi.org/10.1002/hbm.21243

Pressman, S. D., Jenkins, B. N., \& Moskowitz, J. T. (2019). Positive affect and health: What do we know and where next should we go? Annual Review of Psychology, 70, 627-650. https://doi.org/ 10.1146/annurev-psych-010418-102955

Singer, T., \& Lamm, C. (2009). The social neuroscience of empathy. Annals of the New York Academy of Sciences, 1156, 81-96. https://doi.org/10.1111/j.1749-6632.2009.04418.x

Teh, E. J., Yap, M. J., \& Liow, S. (2018). PiSCES: Pictures with social context and emotional scenes with norms for emotional valence, intensity, and social engagement. Behavior Research Methods, 50(5), 1793-1805. https://doi.org/10.3758/s13428-017-0947-x

Vijayakumar, N., Cheng, T. W., \& Pfeifer, J. H. (2017). Neural correlates of social exclusion across ages: A coordinate-based metaanalysis of functional MRI studies. NeuroImage, 153, 359-368. https://doi.org/10.1016/j.neuroimage.2017.02.050

Wesselmann, E. D., Bagg, D., \& Williams, K. D. (2009). "I feel your pain": The effects of observing ostracism on the ostracism detection system. Journal of Experimental Social Psychology, 45, 1308-1311. https://doi.org/10.1016/j.jesp.2009.08.003

Williams K. D. (2007). Ostracism. Annual Review of Psychology, 58, 425-452. https://doi.org/10.1146/annurev.psych.58.110405. 085641

Williams, K. D., Cheung, C. K., \& Choi, W. (2000). Cyberostracism: effects of being ignored over the Internet. Journal of Personality and Social Psychology, 79(5), 748-762. https://doi.org/10.1037// 0022-3514.79.5.748

Younger, J., Aron, A., Parke, S., Chatterjee, N., \& Mackey, S. (2010). Viewing pictures of a romantic partner reduces experimental pain: involvement of neural reward systems. PloS One, 5(10), e13309. https://doi.org/10.1371/journal.pone.0013309

Zhao, J., Mo, L., Bi, R., He, Z., Chen, Y., Xu, F., Xie, H., \& Zhang, D. (2021). The VLPFC versus the DLPFC in Downregulating Social Pain Using Reappraisal and Distraction Strategies. The Journal of Neuroscience, 41(6), 1331-1339. https://doi.org/10.1523/JNEUR OSCI.1906-20.2020

Publisher's note Springer Nature remains neutral with regard to jurisdictional claims in published maps and institutional affiliations. 